\title{
Cryptococcus neoformans isolados de pacientes com AIDS
}

\author{
Cryptocococcus neoformans in patients with AIDS
}

Orionalda de F.L. Fernandes, Théo R. Costa, Márcio R. Costa,

Ailton J. Soares, Ana J.S.C. Pereira e Maria do Rosário R. Silva

Resumo Criptococose é considerada a infecção fúngica sistêmica oportunista mais comum em pacientes com AIDS. Nestes pacientes tem predominado como agente etiológico Cryptococcus neoformans var. neoformans, e muito raramente relata-se $\mathrm{C}$. neoformans var. gattiii, mesmo nas regiões onde se verifica a sua prevalência. Foram estudados 50 pacientes com lesões de criptococose meningoencefálica associada com AIDS. Os isolados foram identificados através de características microscópicas e macroscópicas exibidas em meios de ágar Sabouraud, ágar niger e Christensen. As variedades de C. neoformans foram determinadas pela reação de coloração obtida em meio de L-canavanina glicina-azul de bromotimol (CGB). Em todos os pacientes examinados foram isolados $\mathrm{C}$. neoformans, sendo identificados $\mathrm{C}$. neoformans var. neoformans em 47 isolados e C. neoformans var. gattii em 3. Os resultados encontrados mostram que a criptococose em pacientes com AIDS pode também ser causada por $\mathrm{C}$. neoformans var. gatti, apesar de haver predominância de C. neoformans var neoformans nesta população.

Palavras-chaves: Criptococose. Cryptococcus neoformans. AIDS.

Abstract Cryptococcosis is considered the most frequent fungal systemic opportunist infection in patients with AIDS. C. neoformans var. neoformans infects the patients with AIDS more often than C. neoformans var. gattii which has been rarely isolated from patients with AIDS. Even in endemic regions of $\mathrm{C}$. neoformans var. gattii, the variety of neoformans is still the most common agent diagnosed in cryptococcosis of patients with AIDS. Cerebrospinal fluid (CSF) from fifty patients with neurocryptococcosis associated with AIDS were studied. The fungi were isolated in agar Sabouraud medium, and were identified by microscopic and macroscopic examination by agar Sabouraud, agar niger and Christensen's urea medium. The variety was determined based on a color characteristic reaction on glycine-L-canavanina-bromothymol blue agar. From all of the fifty patients' cerebrospinal fluid (CSF) we were able to recover $\mathrm{C}$. neoformans. The isolates were identified as $\mathrm{C}$. neoformans var. neoformans in 47 patients and as $\mathrm{C}$. neoformans var. gattii. in the 3 remaining patients. These findings suggest that both varieties, gattii and neoformans, are agents of neurocryptococcosis in patients with AIDS.

Key-words: Cryptococosis .Cryptococcus neoformans. AIDS

Infecções fúngicas oportunistas são observadas com grande freqüência em indivíduos imunocomprometidos. Criptococose causada por Cryptococcus neoformans vem assumindo papel relevante por ser considerada uma das micoses mais comuns nos pacientes com síndrome da imunodeficiência humana adquirida (SIDA) produzindo lesões principalmente no sistema nervoso central (SNC), em particular as meninges ${ }^{13141920}$.

A espécie $C$. neoformans possui duas variedades, gattii e neoformans que apresentam

Departamento de Microbiologia Imunologia Parasitologia e Patologia do Instituto de Patologia Tropical e Saúde Pública do Hospital de Doenças Tropicais de Goiânia (HDT) da Universidade Federal de Goiás.

Endereço para correspondência: Prof ${ }^{a}$ Orionalda de Fátima L. Fernandes. Rua 6 № 664, Aptํ 702, Setor Oeste, 74115-070 Goiânia, GO. Fax: 5562 215-2057.

Recebido para publicação em 19/10/98. 
os sorotipos $B-C$ e $A-D$ respectivamente, de acordo com a especificidade antigênica do polissacarídio capsular. Estudos epidemiológicos têm demonstrado diferenças na distribuição geográfica destas variedades ${ }^{10}$. Em pacientes com AIDS, $C$. neoformans var. neoformans tem sido isolado na maioria dos casos diagnosticados de criptococose, mesmo em áreas de clima tropical e subtropical, onde $C$. neoformans var. gattii é relativamente comum ${ }^{211}$.

O presente trabalho tem como principal objetivo verificar a etiologia da criptococose e a variedade predominante nos pacientes com AIDS atendidos no Hospital de Doenças Tropicais de Goiânia, GO, Brasil.

\section{MATERIAL E MÉTODOS}

Foram coletadas amostras de líquido céfalo raquidiano (LCR) de 50 pacientes com AIDS provenientes do Hospital de Doenças Tropicais de Goiânia (HDT) manifestando dor de cabeça, vômito e ocasionalmente febre, sugestivos de criptococose meningo encefálica. As amostras foram submetidas a exame direto com tinta nanquim e cultivados em ágar Sabouraud dextrose com cloranfenicol a temperatura ambiente e a $37^{\circ} \mathrm{C}$ para pesquisa e isolamento dos fungos.
Colônias sugestivas de $C$. neoformans foram repicadas em meios de ágar cenoura batata e ágar niger que contém substratos fenólicos para detectar a atividade da fenol-oxidase, e meio de Christensen para determinar a assimilação da uréia.

As variedades foram determinadas baseandose nas características das cores no meio de L-canavanina-glicina-azul de bromotimol (CGB) segundo Kwon-Chung ${ }^{11}$.

\section{RESULTADOS}

Das 50 amostras de Cryptococcus neoformans, 47 (94\%) foram identificadas como $C$. neoformans var. neoformans e $3(6 \%)$ como $C$. neoformans var. gattii. Os pacientes estudados foram procedentes de diferentes estados do Brasil, sendo que 3 isolados de $C$. neoformans var. gattii e 17 C. neoformans var. neoformans foram de pacientes habitantes do interior de Goiás, 23 isolados foram provenientes de pacientes de Goiânia e os 7 restantes pertencentes a indivíduos dos estados do Tocantins (1), Minas Gerais (2), Pará (3) e São Paulo (1) (Figura 1).

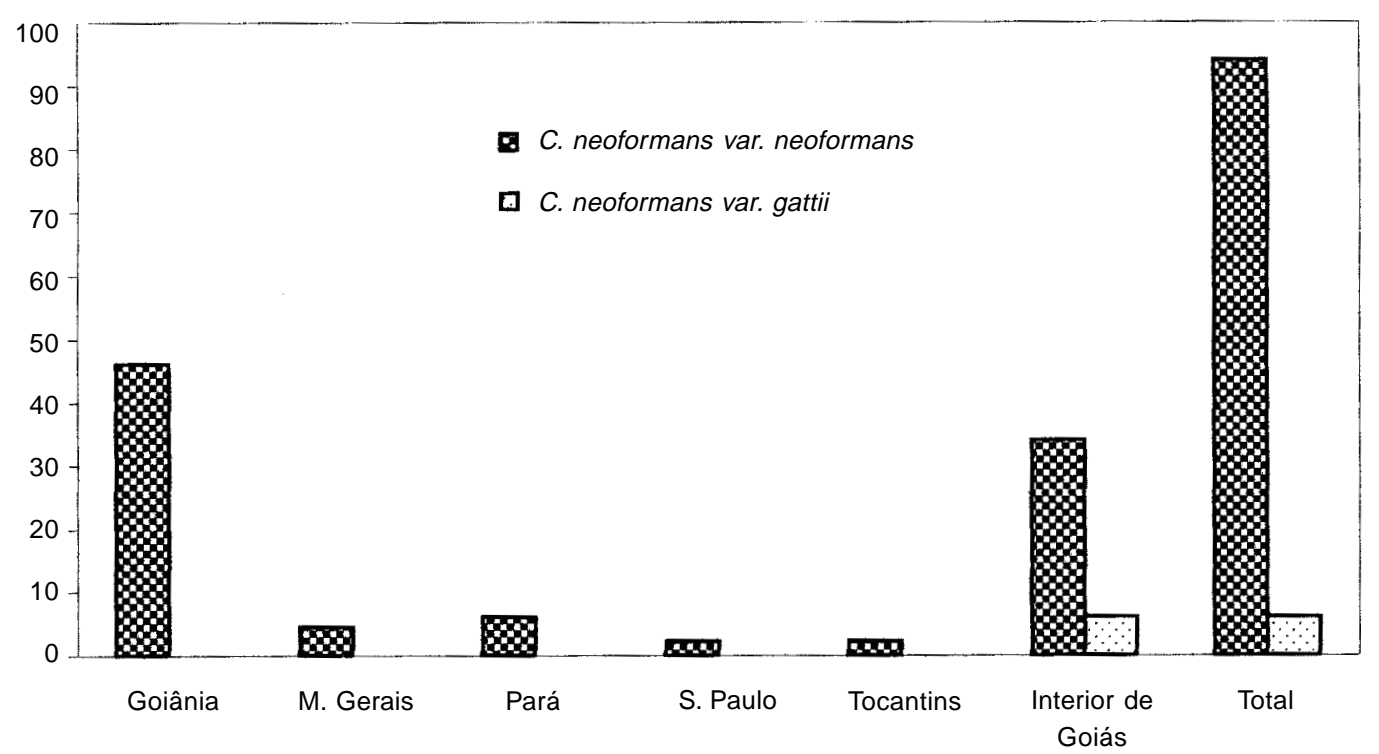

Figura 1 - Procedência regional dos casos de criptococose com seus agentes etiológicos. 
A faixa etária dos pacientes estudados foi de 20 a 60 anos sendo que $56 \%$ possuíam entre 20 e 30 anos. Verificou-se predominância do sexo masculino com $39(78 \%)$ dos casos pertencentes a este sexo. A relação do sexo e idade nos casos de criptococose encontra-se na Tabela 1.

Tabela 1 - Casos de criptococose do sistema nervoso central de pacientes com AIDS relacionados com a idade e sexo.

\begin{tabular}{|c|c|c|c|c|c|c|}
\hline \multirow{3}{*}{$\begin{array}{l}\text { Idade } \\
\text { (anos) }\end{array}$} & \multicolumn{4}{|c|}{ Sexo } & \multirow{2}{*}{\multicolumn{2}{|c|}{ Total }} \\
\hline & \multicolumn{2}{|c|}{$M$} & \multicolumn{2}{|c|}{$F$} & & \\
\hline & $\mathrm{n}^{0}$ & $\%$ & $\mathrm{n}^{0}$ & $\%$ & $\mathrm{n}^{0}$ & $\%$ \\
\hline $20-30$ & 23 & 46 & 5 & 10 & 28 & 56 \\
\hline $31-42$ & 9 & 18 & 4 & 8 & 12 & 24 \\
\hline $41-50$ & 6 & 12 & 1 & 2 & 7 & 14 \\
\hline$>50$ & 1 & 2 & 1 & 2 & 2 & 4 \\
\hline Total & 39 & 78 & 11 & 22 & 50 & 100 \\
\hline
\end{tabular}

\section{DISCUSSÃO}

Todos os pacientes com criptococose estudados no nosso trabalho apresentavam-se com dor de cabeça, vômito e febre ocasionalmente, o que levou a pesquisa de $C$. neoformans no LCR. Esta manifestação clínica é freqüentemente observada em pacientes com AIDS que possuem meningite criptocócia ${ }^{15}{ }^{18}$. Nos Estados Unidos, em 189 casos de criptococose estudados por Chandler et $\mathrm{al}^{5}$, foi verificado uma alta incidência de criptocose meníngea, onde este tipo clínico foi diagnosticado em 167 pacientes.

Os critérios de identificação do agente etiológico de criptococose utilizados em nosso estudo, permitiram mostrar um número elevado de isolados de $C$. neoformans var. neoformans (47/50), enquanto a var. gattii foi isolada em 3 casos. Esta diferenciação de variedades foi observada pelas características exibidas em ágar CGB. A identificação de C. neoformans var. gattii em 3 casos foi considerado de grande importância, visto que mesmo em regiôes endêmicas de criptococose que possui como agente etiológico mais freqüente $C$. neoformans var. gattii, este agente tem sido raramente correlacionado com criptococose do SNC em portadores do vírus da imunodeficiência adquirida ${ }^{14}$. Estudos de vários pesquisadores têm mostrado que a criptococose nos pacientes com AIDS, é geralmente causada por $C$. neoformans var. neoformans independente da área geográfica de sua distribuição ${ }^{16} 17$.

Tem sido postulado que a predominância de C. neoformans var. neoformans em pacientes com AIDS deve-se provavelmente à maior exposição dos indivíduos ao habitat natural deste fungo. Vários trabalhos têm demonstrado que
C. neoformans var. neoformans está correlacionado normalmente a excrementos de fezes de pombos e aves, enquanto C. neoformans var. gattii encontrase associado a árvores de eucaliptos como Eucalyptus camaldulensis e E. tereticornis ${ }^{7}{ }^{8}$. No entanto, em estudos mais recentes têm sido observada a presença de $C$. neoformans nestas fontes naturais, assim como também em ocos de diferentes árvores (Cassia grandis, Senna multijuga, Ficus microcarpa) independente de sua variedade. Segundo estes pesquisadores não existe um relacionamento definido entre o fungo e um tipo específico de habitat ${ }^{312} 13$.

A criptococose foi observada em $78 \%$ dos casos no homem, revelando predominância do sexo masculino sobre o feminino. Duppont et $\mathrm{al}^{6}$ sugerem que pacientes do sexo masculino com AIDS apresentam maior risco à criptococose do que os do sexo feminino. Prevalência do sexo masculino também foi citado por Mitchell et $\mathrm{al}^{14}$ os quais observaram em seu estudo que $80 \%$ dos casos de criptococose do SNC pertenciam a este sexo.

Os pacientes estudados em nosso trabalho possuíam de 20 a 60 anos, sendo que $56 \%$ dos casos apresentavam-se entre 20 e 30 anos. Criptococose em criança com AIDS é menos freqüente, sendo raramente observado. Pinner et $\mathrm{al}^{15}$ relatam $1 \%$ de casos de criptococose em crianças com AIDS.

Os dados encontrados, abrem uma interessante perspectiva sobre a ecologia do Cryptococcus neoformans e sobre a epidemiologia da criptococose, merecendo o prosseguimento de estudos sobre estes temas em pacientes com AIDS. 


\section{REFERÊNCIAS BIBLIOGRÁFICAS}

1. Ansheng L, Nishimura K, Tagushi H, Tanaka R. The isolation of Cryptococcus neoformans from pigeon dropping and serotyping of naturally and clinically sourced isolates in China. Mycopathologia 124:1-5, 1993

2. Bava AJ, Negroni R. Características epidemiológicas de 105 casos de criptococosis diagnosticados em la Repúbliuca Argentina entre 1981-1990. Revista do Instituto de Medicina Tropical de São Paulo 34:335-340, 1992.

3. Bauwens L, Swinne D, De Vroey C, De Meurich W. Isolation of Cyptococcus neoformans var. neoformans in the aviaries of the Antwerp Zoological Garden. Mycosen 29:291-294, 1986.

4. Caiuby J, Rozembaum R, Feijó P, Carvalho A, Peixoto CA, Assis TL, Vohns C, Nogueira AS, Rios-Gonçalves AJ, Associação de criptococose e histoplasmose em pacientes portadores da síndrome da imunodeficiência adquirida. Relato de três casos. Arquivo Brasileiro de Medicina 63:443-445, 1989

5. Chandler FW. Pathology of the mycoses in patients with the acquired immunodeficiency syndrome (AIDS). In: McGinnis MR (ed) Current topics in medical mycology. Springer-Verlag, New York, p.1-23, 1985.

6. Dupont B, Graybill JR, Armstrong D, Laroche R, Touzé JE, Wheat LJ. Fungal Infections in AIDS Patients. Journal of Medical and Veterinary Mycology 30:19-28, 1992.

7. Ellis DH, Pfeiffer TJ. Natural habitat of Cryptococcus neoformans var. gattii. Journal Clinical Microbiology 28:1642-1644, 1990.

8. Emmons CW. Saprophytic source of Cryptococcus neoformans associated with the pigeon (Columbia livia). American Journal of Hygiene 62:227-232, 1955.

9. Kovacs JÁ, Kovacs AA, Polis M. Criptococcosis in the aquired immunodeficiency syndrome. Annals Internal Medicine 103:533-538, 1985.

10. Kwon-Chung KJ, Bennet JE. Epidemiologic differences between the two varieties of Cryptocccus neoformans. American Journal Epidemiology 120:123-130, 1984.

11. Kwon-Chung KJ, Polacheck I, Bennet JE. Improved diagnostic medium for separation of Cryptococcus neoformans var. neoformans (serotypes $A$ and $D$ ) and Cryptococcus neoformans var. gattii (serotype $B$ and $C$ ). Journal Clinical Microbiology 115:535-537, 1982.

12. Lazéra MS, Pires FDA, Camillo-Coura L, Nishikawa MM, Bezerra CCf, Trilles L, Wanke B. Natural habitat of Cryptococcus neoformans var. neoformans in decaying wood forming hollows in living trees. Journal of Medical Veterinary and Mycology 34:127-131, 1996.

13. Lazéra MS, Cavalcante MAS, Nishikawa MM, Wanke B. Cryptococcus neoformans var. gattii evidence for a natural habitat related to decaying wood in pottery tree hollow. Medical Mycology 36:119-122, 1998.

14. Mitchell DH, Sorrell TC, Allworth AM, Heath HC, McGregor AR, Papanaoum K, Richards MJ, Gottlieb T. Criptococcal disease of the CNS in immunocompetent hosts: influence of criptococcal variety on clinical manifestations and outcome. Clinical Infectious Diseases 20:611-616, 1995

15. Pinner RW, Hajjeh RA, Powderly WG. Prospects for preventing cryptococcosis in persons infected with human immunodeficiency vírus. Clinical Infectious Diseases 21:103-107, 1995

16. Rinaldi MG, Drutz DJ, Howell A, Sande MA, Wofsy CB, Hadley, WK. Serotypes of $C$. neoformans in patients with AIDS. Journal Infectious Diseases 153:642, 1986.

17. Rozembaum R, Rios Gonçalves AJ, Wanke B, Caiuby MJ, Clemente H, Lazera MS, Monteiro PC, Londero AT. Cryptococcus neoformans varieties as agents of criptococcose in Brazil Arquivo Brasileiro de Medicina 66:261-263, 1992.

18. Sugar AM. Overview: cryptococcosis in the patients with AIDS. Mycopathologia 114:153-157, 1991.

19. Taborda A, Negroni R, Arechavela A, Robles AM. Criptococosis asociada al SIDA. Estudo retrospectivo de tres terapéuticas antifúngicas en 43 casos. Revista Iberoamericanade Micología 10:10-13, 1993.

20. Zerpa R, Huicho L, Guillén A. Modified india ink preparation for Cryptococcus neoformans in cerebrospinal fluid specimens. Journal of Clinical Microbiology 34:2290-2291, 1996. 